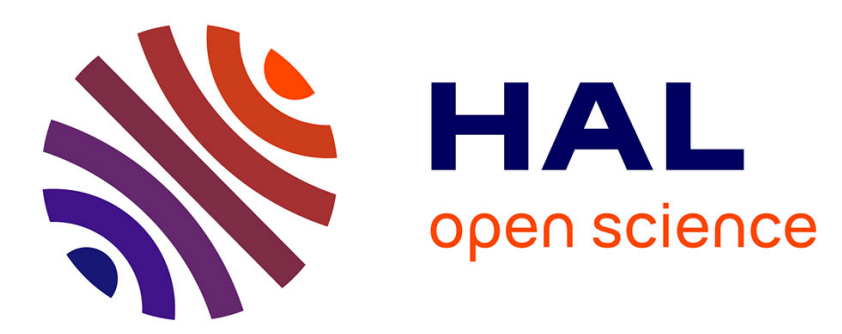

\title{
OFDM/OQAM Blind Equalization Using CNA Approach OFDM/OQAM Blind Equalization Using CNA Approach
}

Vincent Savaux, Jacques Palicot, Faouzi Bader

\section{- To cite this version:}

Vincent Savaux, Jacques Palicot, Faouzi Bader. OFDM/OQAM Blind Equalization Using CNA Approach OFDM/OQAM Blind Equalization Using CNA Approach. IEEE Transactions on Signal Processing, 2016, 64 (9), pp.2324-2333. 10.1109/TSP.2016.2519000 . hal-01267300

\section{HAL Id: hal-01267300 \\ https://hal.science/hal-01267300}

Submitted on 5 Feb 2016

HAL is a multi-disciplinary open access archive for the deposit and dissemination of scientific research documents, whether they are published or not. The documents may come from teaching and research institutions in France or abroad, or from public or private research centers.
L'archive ouverte pluridisciplinaire HAL, est destinée au dépôt et à la diffusion de documents scientifiques de niveau recherche, publiés ou non, émanant des établissements d'enseignement et de recherche français ou étrangers, des laboratoires publics ou privés. 


\title{
OFDM/OQAM Blind Equalization Using
}

\author{
CNA Approach
}

\author{
Vincent Savaux, Faouzi Bader, Jacques Palicot
}

\begin{abstract}
This paper deals with blind equalization based on the constant norm algorithm (CNA) in orthogonal frequency division multiplexing/offset quadrature amplitude modulation (OFDM/OQAM). The CNA is designed to fit the complex square constellations with high order. As a consequence, the received signal is reshaped in order to change the real OQAM symbols into complex ones. Although it increases the intrinsic interferences of the OFDM/OQAM signal, it is proved by analysis and through simulations that the CNA with proposed reshaped signal achieves better mean square error (MSE) performance than CMA applied to the received OFDM/OQAM signal. Furthermore, a suboptimal initialization strategy is proposed, which ensures a high convergence speed. The behavior of the proposed blind equalization method is analyzed under synchronization mismatch as well as in time-varying propagation environment.
\end{abstract}

\section{Index Terms}

Blind equalization, OFDM/OQAM, Iterative Methods, CMA, CNA, impulse noise cancellation.

\author{
Manuscript submitted July 2015 \\ Vincent Savaux, Faouzi Bader, and Jacques Palicot are with the Signal, Communication \& Embedded Electron- \\ ics (SCEE) team/IETR, CentraleSupélec, Rennes, France (e-mails: vincent.savaux,jacques.palicot@centralesupelec.fr, \\ faouzi.bader@supelec.fr Phone: +33 299844536; Fax:+33 299844599.)
}

This work is supported by the project PROFIL (Evolution of the Wideband Professional Mobile Radio Based on the Filter Bank MultiCarrier Modulation) funded by the French national research agency with grant agreement code: ANR-13INFR-0007-03, and ICT EC-funded project Newcom\# with code: FP7-IC-318306.

EDICS: SPC-CEST 


\section{OFDM/OQAM Blind Equalization Using}

\section{CNA Approach}

\section{INTRODUCTION}

Filter bank multicarrier (FBMC), and in particular the orthogonal frequency division multiplexing/Offset quadrature amplitude modulation (OFDM/OQAM) is a good alternative to the OFDM for systems coexistence and spectrum sharing as it achieves better spectral containment [1], [2]. Furthermore, it provides higher useful bit rate as it does not require any cyclic prefix addition. However, the good properties of OFDM/OQAM are due to subcarrier functions that are only orthogonal in the real field. As a consequence, the presence of imaginary intrinsic interference and the complex channel frequency response lead to develop some equalization methods specific to OFDM/OQAM.

As in any modulation scheme, the channel estimation and equalization techniques in OFDM/OQAM can be classified into two main categories:

i) the data-aided methods, in which pilots are multiplexed in the data stream and the signal is equalized using the estimated channel (see [3]-[6] for data aided estimation methods). The usual zero forcing (ZF) and minimum mean square error (MMSE) equalizer are presented in a FBMC context in [7], and the authors of [8] propose to split the phase and the modulus equalization. In [9], the topic of channel equalization has been extended to FBMC modulation scheme in multiple antennas systems. ii) the blind techniques, which use only few features of the signal to invert the channel (see [10]-[12]).

Although data-aided methods are simple to implement and widely employed, they may reduce the spectral efficiency of the transmitted signal. On the other hand, blind techniques have not been so deeply studied in OFDM/OQAM context, and the topic remains a pending challenge.

In [10], the cyclostationarity induced by the use of the OFDM/OQAM pulse shape is exploited in order to perform a second order blind equalization. However, this technique has a high complexity, 
and very long data records have to be stored to obtain good estimates of the second-order statistics of the signal. The more "classical" constant modulus algorithm (CMA) has been adapted to FBMC (cosine modulated filter bank) in [11], and its convergence behavior has been analyzed in [12]. The idea behind CMA applied in OFDM/OQAM is to iteratively update the algorithm by comparing the real part of the output of the equalizer with a given real constant that depends on the constellation size. However, it is worth noting that CMA is equivalent to Sato's algorithm [13], the multimodulus algorithm (MMA) [14], and the constant norm algorithm (CNA) [15], due to the use of real transmitted OQAM symbols. Therefore, this limits the choice of the cost function to those that are adapted to real constellation, whereas numerous cost functions adapted to different complex constellations have been proposed in the scientific literature [15]-[17].

In this paper, it is proposed to reshape the received signal by summing two consecutive symbols such that, from the equalizer point of view, the received symbols are complex. This provides an additional degree of freedom regarding the choice of the cost function of the update algorithm. As a consequence, we take advantage of these reshaped complex symbols to carry out a blind equalization using the CNA proposed in [15], which has proven to be particularly adapted to square constellations. This algorithm can be seen as a generalization of the CMA, where the modulus is substituted by a norm which is determined as a function of the considered constellation. The performance of the proposed CNA adapted to OFDM/OQAM is analyzed, and it is proved that the CNA achieves better performance than the CMA.

The rest of the paper is organized as follows: the blind OFDM/OQAM receiver structure is presented in Section II. The proposed CNA using a reshaped received signal is developed in Section III. Section IV provides simulations results that show the performance of the proposed blind equalization techniques. Finally, we draw our conclusions in Section V.

\section{BLIND OFDM/OQAM RECEIVER}

The blind OFDM/OQAM receiver is presented in Fig. 1. The demodulator consists of a serial to parallel converter (denoted by S/P), and an analysis filter bank (AFB). The latter is composed of i) 
the polyphase network (PPN) that contains a set of digital filters, and ii) the fast Fourier transform (FFT) of size $M$ ( $\mathrm{M}$ is also the number of subcarriers).

In the following expression, the receiver is considered to be perfectly synchronized with the transmitter in both time and frequency domains, and the channel delay is assumed to be short compared to the length of the used prototype filter, in such a way that the output of the AFB described in Fig. 1-(a) can be written as

$$
y_{m, n}=H_{m, n} x_{m, n}+\underbrace{j \sum_{(p, q) \in \Omega} H_{p, q} x_{p, q}<g>_{m, n}^{p, q}}_{I_{m, n}}+w_{m, n},
$$

where the subscripts " $m, n "$ denote the frequency-time position. $H_{m, n}$ is the channel frequency response (CFR), $w_{m, n}$ is the complex Gaussian noise sample with circular covariance matrix $\mathbf{R}_{w}$. Note that for convenience, this matrix is commonly approximated by $\sigma^{2} \mathbf{I}$, where $\sigma^{2}$ is the noise level, and $\mathbf{I}$ is the identity matrix. $x_{m, n}$ is the real OQAM symbol that is carried by the prototype filter $g$ which is given by

$$
g_{m, n}[i]=g\left(i-n \frac{M}{2}\right) e^{\frac{2 j \pi m}{M}\left(i-\frac{L_{f}-1}{2}\right)} e^{j \phi_{m, n}}
$$

where the phase term is $\phi_{m, n}=(\pi / 2)(m+n)+m n \pi$ as defined in [18]. It should be noted that the real symbols $x_{m, n}$ are transmitted with a period $\frac{M}{2}$ twice shorter than that of OFDM signal where symbols are complex. Therefore, the data rate in OFDM/OQAM is the same as in OFDM. The specificity of the OFDM/OQAM modulation scheme lies in the presence of interferences denoted by $I_{m, n}$ in (1), which is due to the used prototype filter $g$. The term $j<g>_{m, n}^{p, q}$ in (1) is called "intrinsic interference" (according to the definition in [6]), and is defined as the scalar product

$$
\sum_{i} g_{m, n}[i] g_{p, q}^{*}[i] d t=\left\{\begin{array}{ll}
1, & \text { if }(p, q)=(m, n) \\
j<g>_{p, q}^{m, n}, & \text { else }
\end{array} .\right.
$$




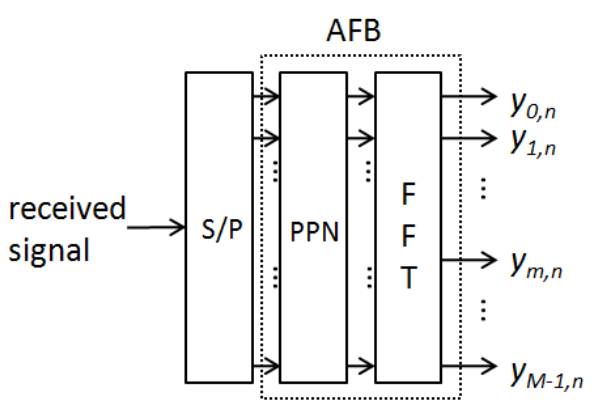

(a) OFDM/OQAM receiver.

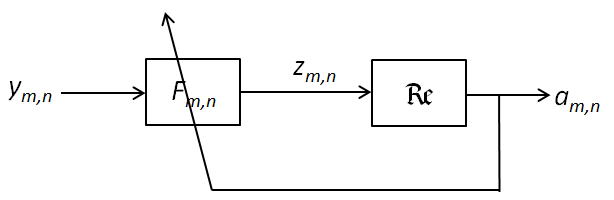

(b) Per-carrier blind equalizer in

Fig. 1. Blind OFDM/OQAM receiver structure with (a) AFB, and (b) blind equalizer.

In this paper, the Bellanger's filter is employed, but other filters with self interference effect could be used. This filter is also known as "PHYDYAS filter", and its frequency response can be expressed as (see [19] for details)

$$
G[f]=\sum_{k=-(K-1)}^{K-1} G_{k} \frac{\sin \left(\pi\left(f-\frac{k}{L_{f}}\right) L_{f}\right)}{L_{f} \pi\left(f-\frac{k}{L_{f}}\right)},
$$

where $G_{0}=1, G_{1}=0.971960, G_{2}=1 / \sqrt{2}$, and $G_{3}=0.235147$ according to [19], and $G_{k}=G_{-k}$. $L_{f}$ is the length of the filter defined as $L_{f}=K M$, where $K$ is called overlapping factor. As mentioned in [6]-eq. (13), the weights $\left\langle g>_{m, n}^{p, q}\right.$ of any well-chosen prototype filter have a symmetrical property as

$$
\begin{array}{ccc}
(-1)^{p} a & -b & (-1)^{p} a \\
-(-1)^{p} c & 1 & (-1)^{p} c \\
(-1)^{p} a & b & (-1)^{p} a
\end{array}
$$

where the weight 1 corresponds to the position $(m, n)$, and where $a, b$, and $c$ values depend on $M$ and $K$. Note that, due to (5), the noise component $w_{m, n}$ cannot be white. However, the assumption of "whiteness" of the noise in OFDM/OQAM has proven to be accurate, such as in [20].

The pulse shape $g$ allows the OFDM/OQAM signal to have a good spectral containment, but it is orthogonal only in the real field. Thus, the interference term $I_{m, n}$ is purely imaginary if $H_{m, n}=1$, and 
therefore the real OQAM symbols can be recovered by $\hat{x}_{m, n}=\mathfrak{R e}\left\{y_{m, n}\right\}$. However, the presence of the channel gain $H_{m, n} \in \mathbb{C}$ induces complex interferences from all the symbols at positions $(p, q) \in \Omega$, where $\Omega$ is the set of frequency-time positions that surround $(m, n)$. As a consequence, the distortion due to the channel must be canceled in order to recover the real orthogonality between subcarriers and symbols.

It is worth noting that in practice, the received signal $y_{m, n}$ in (1) is also distorted by some residual channel frequency offset (CFO) and time synchronization mismatch [21]-[23]. In this paper, it is assumed that the misalignment between the carrier frequencies generated at the transmitter and the receiver can be neglected in comparison with the subcarrier distance. However, the time synchronization mismatch induces a phase rotation after the demodulation stage, in such a way that the received signal should be rewritten $y_{m, n} e^{-2 j \pi \frac{\tau_{k} m}{M}}$ where $\tau_{k}$ is the time shift.

In this paper, the signal is blindly equalized, which maximize its spectral efficiency since no pilot is used. Some techniques have been proposed in the literature for both single-carrier as in [16], [24], [25] and [15] and in multicarrier systems as OFDM/OQAM [10]-[12]. The basic idea behind the blind equalization is to iteratively update the equalizer coefficient $F_{m, n}$ by using only few features of the signal. In the OFDM/OQAM modulation scheme, this iterative process is carried out on each subcarrier $m \in \llbracket 0, M-1 \rrbracket$, as described in Fig. 1-(b). The corresponding output of the equalizer can be expressed as

$$
a_{m, n}=\mathfrak{R e}\left(F_{m, n} y_{m, n}\right) .
$$

The optimal coefficient $F_{m, n}^{o p t}$ can be obtained on each subcarrier by solving the optimization problem defined as

$$
F_{m, n}^{o p t}=\min _{F_{m, n}} J\left(a_{m, n}\right)
$$

where $J$ is a given cost function that needs to be defined. In the rest of the paper, the minimization step in (7) is achieved by means of the usual stochastic gradient method as in [11], [24] and [15], 
and which can be expressed as

$$
F_{m, n+1}=F_{m, n}-\mu \phi\left(a_{m, n}\right),
$$

where $\mu$ is the step-size parameter of the update algorithm, and

$$
\phi\left(a_{m, n}\right)=\frac{\partial J\left(a_{m, n}\right)}{\partial F_{m, n}^{*}} .
$$

It should be noticed that $a_{m, n}=\mathfrak{R e}\left(F_{m, n} y_{m, n}\right)$ is not an holomorphic function. Therefore, the Wirtinger's derivation [26] is used in (9) instead of the usual complex derivation. It has been demonstrated in [11] that the constant modulus algorithm (CMA) proposed by Godard in [24] is suitable to OFDM/OQAM. Furthermore, note that for the same reason, the modified CMA (MCMA) in [16] (also called multimodulus algorithm (MMA) in [14]) is exactly the same as CMA in the OFDM/OQAM modulation scheme. The cost function of CMA is written as

$$
J\left(a_{m, n}\right)=E\left\{\left(\left|a_{m, n}\right|^{2}-\gamma\right)^{2}\right\}
$$

where $\gamma$ is a real-valued constant that is defined hereafter ${ }^{1}$. The update algorithm can be rewritten by substituting (10) into (8) as

$$
F_{m, n+1}=F_{m, n}-2 \mu\left(\left|a_{m, n}\right|^{2}-\gamma\right) \frac{\partial\left|a_{m, n}\right|^{2}}{\partial F_{m, n}^{*}}
$$

The modulus $\left|a_{m, n}\right|^{2}$ can be rewritten as

$$
\begin{aligned}
\left|a_{m, n}\right|^{2} & =\frac{1}{4}\left(F_{m, n} y_{m, n}+\left(F_{m, n} y_{m, n}\right)^{*}\right)^{2} \\
& =\frac{1}{4}\left(F_{m, n} y_{m, n}\right)^{2}+\frac{1}{4}\left(\left(F_{m, n} y_{m, n}\right)^{*}\right)^{2} \\
& +\frac{1}{2} F_{m, n} y_{m, n}\left(F_{m, n} y_{m, n}\right)^{*} .
\end{aligned}
$$

\footnotetext{
${ }^{1}$ Note that the CMA can be written in a normalized manner, by factorizing by $\gamma$.
} 
Furthermore, the Wirtinger's derivative leads to $\frac{\partial z}{\partial z^{*}}=\frac{\partial z^{*}}{\partial z}=0$ for any $z \in \mathbb{C}$ (see Theorem 2.5 in [26]), then the substitution of (12) into (11) yields

$$
F_{m, n+1}=F_{m, n}-2 \mu y_{m, n}^{*} a_{m, n}\left(\left|a_{m, n}\right|^{2}-\gamma\right)
$$

As indicated in [24], the optimal value of the constant $\gamma$ must be chosen as

$$
\gamma=\frac{E\left\{|x|^{4}\right\}}{E\left\{|x|^{2}\right\}}
$$

In the following, we propose two alternatives to CMA for blind equalization in OFDM/OQAM. Based on the results obtained for single-carrier modulation scheme in [15], we adapt the CNA to OFDM/OQAM systems in hereafter Section III.

\section{AdAPTING THE CNA IN OFDM/OQAM}

The basic idea behind the CNA is to use a $k$-norm, $k>2$, instead of the modulus in the cost function of the equalizer (10). It has been proved in [15] that CNA achieves better performance than CMA for square constellations as 16-QAM, since CMA is adapted for constant modulus constellations. Furthermore, it has been shown in [15] that for each square constellation corresponds a given $k$ norm that maximizes the performance of the equalizer. However, the transmitted symbols in (1) are real, therefore the use of CNA in OFDM/OQAM requires some adaptations. Principally, the received signal in (1) must be redesigned and the symbols combined in order to highlight complex transmitted symbols. Since CNA is suitable for high order constellations, we consider throughout this section that the real OQAM symbols $x_{m, n}$ are randomly chosen among the set $\{-3,-1,1,3\}$, which corresponds to the real part of 16-QAM. The aims of this section are twofold: i) presenting the reshaping of the input signal $y_{m, n}$ in (1) in order to highlight complex-valued symbols $\tilde{x}_{m, n}$, and ii) proving that carrying out the CNA using the reshaped inputs $\tilde{x}_{m, n}$ lieds to better performance than using CMA with real inputs $x_{m, n}$. 


\section{A. Rewriting the Received Signal with Complex Input Symbols}

Since the transmitted symbols $x_{m, n}$ are real, the first step before applying the CNA consists of converting the received symbols into complex data. To achieve this, the following procedure is carried out:

1) Build $y_{m, n}^{c}$ defined as the sum of two consecutive received symbols $y_{m, n}$ and $y_{m, n+1}$ as

$$
\begin{aligned}
y_{m, n}^{c}= & j y_{m, n}+y_{m, n+1} \\
= & H_{m, n} j x_{m, n}+H_{m, n+1} x_{m, n+1} \\
& +j I_{m, n}+I_{m, n+1}+j w_{m, n}+w_{m, n+1} .
\end{aligned}
$$

Note that $H_{m, n}=H_{m, n+1}$ in (15), which leads to further developments.

2) $y_{m, n}^{c}$ can be rewritten by substituting (1) into (15) as

$$
\begin{aligned}
y_{m, n}^{c}= & H_{m, n}\left(j x_{m, n}\left(1+<g>_{m, n+1}^{m, n}\right)\right. \\
& \left.+x_{m, n+1}\left(1-<g>_{m, n}^{m, n+1}\right)\right) \\
& -\underbrace{\sum_{\substack{(p, q) \in \Omega \\
q \neq n+1}} H_{p, q} x_{p, q}<g>_{m, n}^{p, q}}_{\tilde{I}_{m, n}} \\
& +\underbrace{j \sum_{\substack{(p, q) \in \Omega \\
q \neq n}} H_{p, q} x_{p, q}<g>_{m, n+1}^{p, q}}_{\tilde{I}_{m, n+1}} \\
& +j w_{m, n}+w_{m, n+1} .
\end{aligned}
$$

It must be emphasized that (16) holds since the channel is assumed to be static. The effect of channel variations on the accuracy of (16) will be investigated in Section IV. The symmetry property of weights $\langle g\rangle_{m, n}^{p, q}$ given in (5) yields $\left\langle g>_{m, n+1}^{m, n}=-\langle g\rangle_{m, n}^{m, n+1}\right.$, and therefore we can define $\tilde{y}_{m, n}$ from $y_{m, n}^{c}$ in (16) as 


$$
\begin{aligned}
\tilde{y}_{m, n}= & \frac{y_{m, n}^{c}}{1+<g>_{m, n+1}^{m, n}} \\
& =H_{m, n} \underbrace{x_{m, n}}_{\tilde{x}_{m, n}\left(j x_{m, n}+x_{m, n+1}\right)} \\
& \underbrace{\frac{-\tilde{I}_{m, n}+\tilde{I}_{m, n+1}}{1+<g>_{m, n+1}^{m, n}}}_{\tilde{I}_{m, n}^{r}}+\underbrace{\frac{j w_{m, n}+w_{m, n+1}}{1+<g>_{m, n+1}^{m, n}}}_{\tilde{w}_{m, n}},
\end{aligned}
$$

where $\tilde{x}_{m, n}$ is a 16-QAM symbol.

It is worth noticing that the redesigned received signal in (17) is exactly equivalent to the transmission of complex 16-QAM symbols $\tilde{x}_{m, n}$ over the channel $H_{m, n}$, with additive interference $\tilde{I}_{m, n}^{r}$ and non-white Gaussian noise $\tilde{w}_{m, n}$. Therefore, the CNA can now be employed as blind equalizer whose input $z_{m, n}$ is defined as

$$
z_{m, n}=j a_{m, n}+a_{m, n+1}
$$

where $a_{m, n}$ and $a_{m, n+1}$ are the outputs of the equalizer given by (6). The specific update algorithm for $F_{m, n}$ is described in the hereafter Section III-B. The overall principle of the blind equalizer using CNA adapted to OFDM/OQAM is depicted in Fig. 2.

\section{B. Deriving CNA in OFDM/OQAM}

The cost function of the CNA can be formulated in a general expression as

$$
J\left(z_{m, n}\right)=\frac{1}{a b}\left|\left\|z_{m, n}\right\|^{a}-\gamma\right|^{b}
$$

where $\|$.$\| is a norm defined on \mathbb{C}$, and $a$ and $b$ are two parameters that give two degrees of freedom to the algorithm. In the following, it will be assumed that ${ }^{2} a=b=2$. Since $x_{m, n}$ is taken from a 16-QAM constellation, the real positive constant $\gamma$ should be rewritten as

\footnotetext{
${ }^{2}$ This choice is motivated, once again, by the difficulty of setting a proper step-size $\mu$ value when $a$ and $b$ increase.
} 


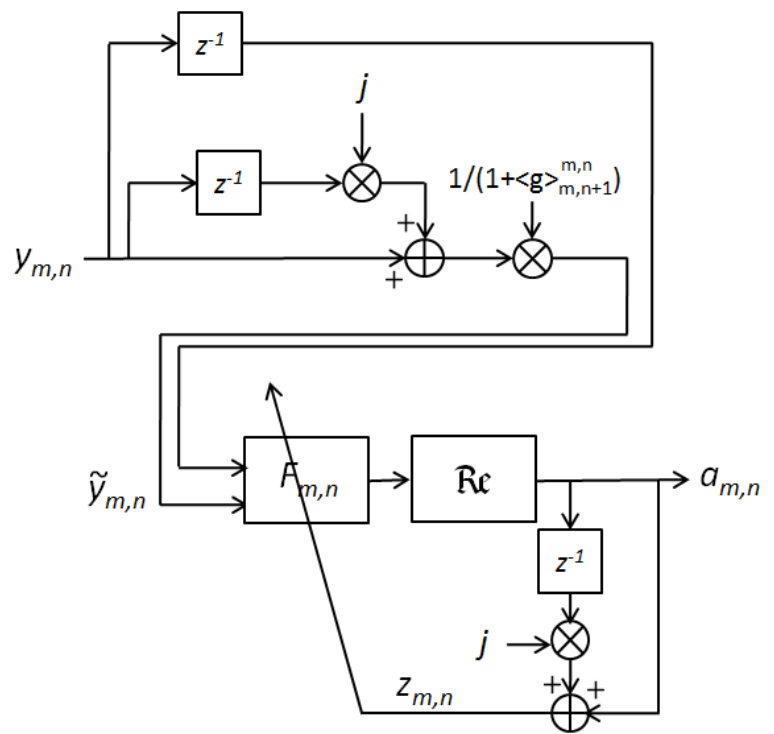

Fig. 2. Proposed blind equalizer using CNA, adapted to OFDM/OQAM system.

$$
\gamma=\frac{E\left\{\left\|x_{m, n}\right\|^{4}\right\}}{E\left\{\left\|x_{m, n}\right\|^{2}\right\}}=13.2
$$

The $k$-norm family $\|$.$\| considered in this paper can be formulated for any complex z$ on the plane $\mathbb{C}$ as

$$
\|z\|_{k}=\sqrt[k]{|\operatorname{Re}\{z\}|^{k}+|\operatorname{Im}\{z\}|^{k}}
$$

Note that the CNA reduces to the CMA when $k=2$. It has been demonstrated in [15] that the case $k=6$ (denoted by CNA-6) is very suitable for 16-QAM constellation. According to this result, we hereafter employ the CNA-6 whose update algorithm can be adapted to OFDM/OQAM using (17) as

$$
\begin{aligned}
F_{m, n+1}= & F_{m, n}-\mu\left(\left\|z_{m, n}\right\|_{6}^{2}-\gamma\right) \\
& \times \frac{\mathfrak{R e}\left\{z_{m, n}\right\}^{5}+j \mathfrak{I m}\left\{z_{m, n}\right\}^{5}}{\left\|z_{m, n}\right\|_{6}^{4}} \tilde{y}_{m, n}^{*} .
\end{aligned}
$$

The steady-state performance of any constant norm-based equalizers can be characterized by the average distance $E\{l\}$ between the constellation symbols and the ball defined in (10) and (19) (see 


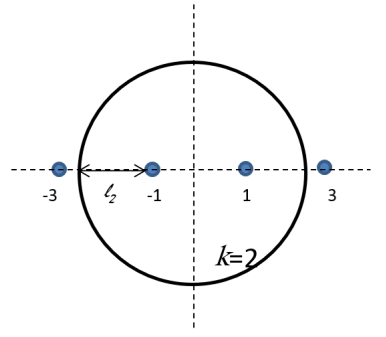

(a) Balls of CMA , compared with the symbols of the constellation $\{-3,-1,1,3\}$.

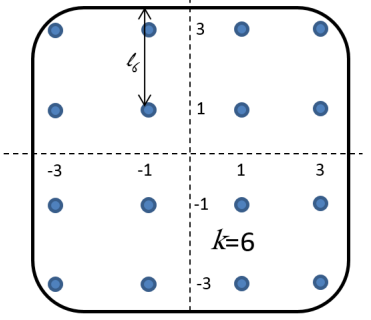

(b) Balls of CNA-6, compared with the symbols of a 16QAM.

Fig. 3. Balls of (a) CNA-2 (CMA) in (10) compared with $\{-3,-1,1,3\}$, and (b) CNA-6 in (22) compared 16-QAM.

details [27]). Fig. 3 shows the balls of the modulus and the 6-norm compared with the 16-QAM in 3(b), and with the corresponding real OQAM symbols $\{-3,-1,1,3\}$ in 3 -(a). It can be deduced from Fig. 3 that $E\left\{l_{6}\right\}=1.0586>E\left\{l_{2}\right\}=1$. The developments leading to this results are provided in Appendix A. As a consequence, the performance of the CNA-6 using the proposed reshaped symbols $\tilde{y}_{m, n}$ in (17) should be lower than that of CMA using $y_{m, n}$ in (1). However, the distance $E\{l\}$ does not take into account the interferences, and therefore a deeper analysis must be undertaken in order to show the influence of the interferences on the performance of the CMA and CNA-6.

\section{Performance Analysis of CNA-6 Adapted to OFDM/OQAM}

The proposed development leading to $\tilde{y}_{m, n}$ in (17) induces some changes on the noise variance and on the energy of the interferences. Thus, the variance $\tilde{\sigma}^{2}$ of the noise $\tilde{w}_{m, n}$ can be expressed as

$$
\tilde{\sigma}^{2}=E\left\{\left|\tilde{w}_{m, n}\right|^{2}\right\}=\frac{2 \sigma^{2}}{\left|1+<g>_{m, n+1}^{m, n}\right|^{2}} .
$$

It should be emphasized that $\tilde{\sigma}^{2}$ in (23) depends on the chosen filter $g$, and thus, the noise level could be either higher or lower than the original noise variance $\sigma^{2}$. In the considered case of PHYDYAS's filter, the noise variance is reduced as $\tilde{\sigma}^{2}=0.8172 \sigma^{2}$.

Fig. 4 depicts the probability density functions (PDFs) of the interferences $\mathfrak{I m}\left(I_{m, n}\right), \mathfrak{R e}\left(\tilde{I}_{m, n}^{r}\right)$, and $\mathfrak{I m}\left(\tilde{I}_{m, n}^{r}\right)$ in (1) and (17), respectively. These have been obtained thanks to an histogram over 


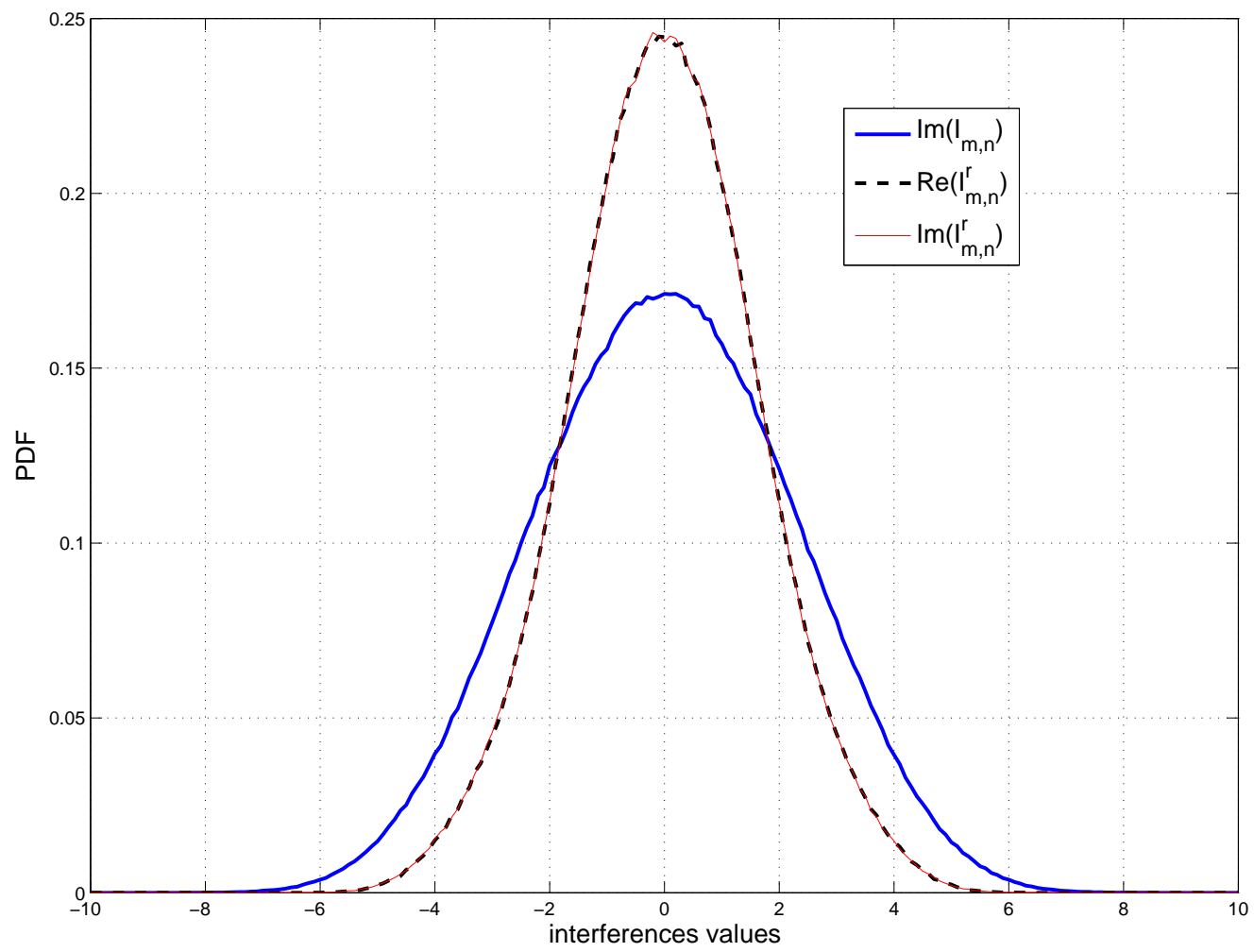

Fig. 4. Distributions of the interferences $I_{m, n}$ in (1) and $\tilde{I}_{m, n}^{r}$ in (17).

10000 simulation runs. One can observe that $\mathfrak{I m}\left(I_{m, n}\right)$ is purely real (by definition) with a variance $\sigma_{I}^{2}=5$, whereas $\tilde{I}_{m, n}^{r}$ is complex with a variance $\sigma_{\tilde{I}}^{2}=5.387$. Knowing that $x_{m, n} \in\{-3,-1,1,3\}$ whereas $\tilde{x}_{m, n}$ are 16-QAM symbols, we can conclude that the signal to interference ratios related to the CMA and the CNA-6 are such that

$$
\frac{E\left\{\left|x_{m, n}\right|^{2}\right\}}{\sigma_{I}^{2}}<\frac{E\left\{\left|\tilde{x}_{m, n}\right|^{2}\right\}}{\sigma_{\tilde{I}}^{2}} .
$$

We can deduce from (24) that the signal used for the CNA-6 should be less sensitive to interference than that used for the CMA, even if $\sigma_{\tilde{I}}^{2}>\sigma_{I}^{2}$.

In addition to (24), the performances of the equalizers can be compared in terms of excess mean square error (EMSE), which assesses the noise of the equalizer in the steady-state. From [15], the EMSEs of CMA and CNA-6 denoted by $\Gamma_{C M A}$ and $\Gamma_{C N A 6}$ can be approximated as in (25) and (26) 


$$
\Gamma_{C N A 6} \approx \mu P_{\tilde{y}} \frac{E\left\{\left(\left\|z_{o p}\right\|_{6}^{2}-\gamma\right)^{2}\left(\mathfrak{R e}\left(z_{o p}\right)^{10}+\mathfrak{I m}\left(z_{o p}\right)^{10}\right) /\left\|z_{o p}\right\|_{6}^{8}\right\}}{E\left\{\left[\left(4 \gamma-2\left\|z_{o p}\right\|_{6}^{2}\right)\left(\mathfrak{R e}\left(z_{o p}\right)^{10}+\mathfrak{I m}\left(z_{o p}\right)^{10}\right)+5\left\|z_{o p}\right\|_{6}^{6}\left(\left\|z_{o p}\right\|_{6}^{2}-\gamma\right)\left(\mathfrak{R e}\left(z_{o p}\right)^{4}+\mathfrak{I m}\left(z_{o p}\right)^{4}\right)\right] /\left\|z_{o p}\right\|_{6}^{10}\right.}
$$

$$
\Gamma_{C M A} \approx \mu P_{y} \frac{E\left\{\left|a_{o p}\right|^{6}-2 \gamma\left|a_{o p}\right|^{4}+\gamma^{2}\left|a_{o p}\right|^{2}\right\}}{E\left\{4\left|a_{o p}\right|^{2}\right\}-2 \gamma}
$$

where $a_{o p}$ and $z_{o p}$ are the perfectly equalized symbols (i.e. when $F_{m}^{o p t} H_{m}=1$ is assumed) in (6) and (18), respectively, and $P_{y}$ and $P_{\tilde{y}}$ are the powers of the corresponding input signals $y_{m, n}$ and $\tilde{y}_{m, n}$, respectively.

It is worth noting that $\Gamma_{C M A}$ in (25) and $\Gamma_{C N A 6}$ in (26) are linear functions of $P_{y}$ and $P_{\tilde{y}}$ respectively. Since these inputs powers are functions of the interferences, it is possible to compare the achieved $\Gamma_{C M A}$ and $\Gamma_{C N A 6}$ values in function of the interference power, as depicted in Fig. 5. The step-size $\mu$ value has been arbitrarily set equal to $4.10^{-4}$ (in practice, the step-size parameter of CMA is larger than CNA's, as described in Section IV). Note that different $\mu$ values would only lead to a change in the slope of the curves. It can be observed that $\Gamma_{C N A 6}<\Gamma_{C M A}$ for any interference power value. It reflects the fact that the CNA-6 with the proposed solution described in Fig. 2 achieved better performance than CMA using the real OQAM symbols as in [11]. This theoretical performance results will be confirmed through simulations in Section IV.

Beyond the application of the blind equalizer using CNA-6 in OFDM/OQAM, the reshape of complex symbols as described in Fig. 2 opens new perspectives for blind equalization in filter bankbased modulations, as specific cost functions (not limited to the real field) can now be used and investigated.

\section{Proposed Initialization Strategy}

Since the proposed blind equalizer avoids the convergence of the coefficients $F_{m, n}$ toward local minima, it is not mandatory to choose a very low initialization value $F_{m, 0}$ as proposed in [11], 


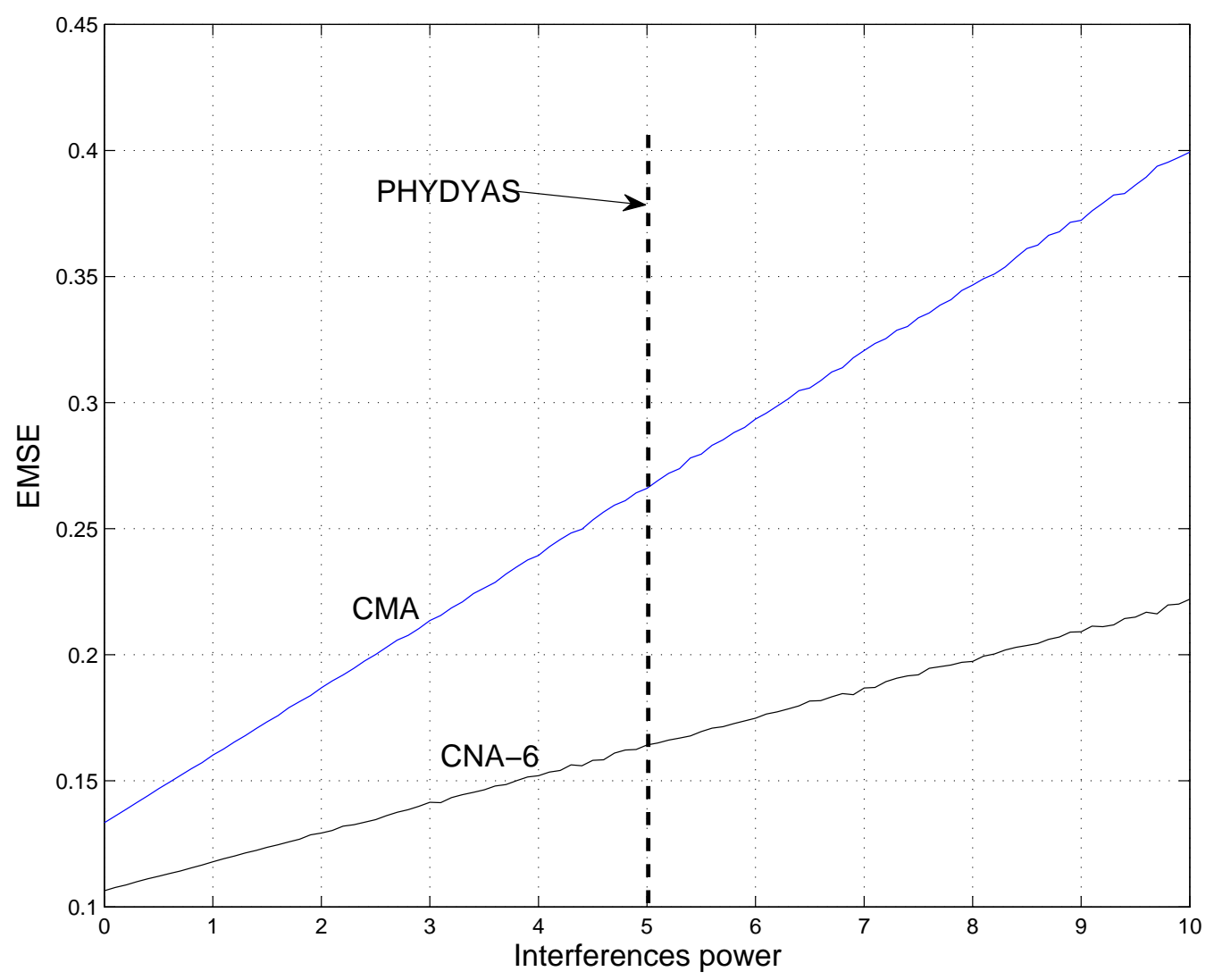

Fig. 5. EMSE versus interference power, comparison between CMA and CNA-6.

[12]. This solution may especially appear not adapted for channels with deep fading as the optimal coefficients $\left|F_{m, n}^{o p t}\right|$ have high values, which should lead to a long convergence time. It has been demonstrated in [28] that a sub-optimal initialization value (in the mean square error sense) can be assessed in order to take into account the channel value for each frequency position $m$. To achieve this, the coefficients can be defined for $n=0$ and for any $m \in\{0,1, . ., M-1\}$ as

$$
F_{m, 0}^{s-o p t}=\sqrt{\frac{E\left\{x_{m, 0}^{2}\right\}}{\left|y_{m, 0}\right|^{2}+\alpha}},
$$

where $\alpha$ is a real constant that avoids the divergence of $F_{m, 0}$ when $y_{m, 0}$ is very close to zero. In practice, $\alpha$ is chosen such that $\alpha<<1$.

The sub-optimal nature of the initialization $F_{m, 0}^{s-o p t}$ is shown hereafter, and compared with the 
solution $\left|F_{m, 0}\right|<<1$ proposed in [11], [12]. The mean error between the square moduli of the inverse of $F_{m, 0}^{s-o p t}$ and $H_{m, 0}$ is defined as

$$
\epsilon_{F}=E\left\{\frac{1}{\left|F_{m, 0}^{s-o p t}\right|^{2}}-\left|H_{m, 0}\right|^{2}\right\}
$$

Note that the noise samples $w_{m, 0}$ have a variance $\sigma^{2}$, and are uncorrelated with $x_{m, 0}, H_{m, 0}$, and $I_{m, 0}$. Therefore, the mean error $\epsilon_{F}$ in (28) can be rewritten by substituting (1) into (27) and (28) as

$$
\begin{aligned}
\epsilon_{F} & =E\left\{\frac{\left|y_{m, 0}\right|^{2}+\alpha}{E\left\{x_{m, 0}^{2}\right\}}-\left|H_{m, 0}\right|^{2}\right\} \\
& =E\left\{\frac{\left|x_{m, 0} H_{m, 0}+I_{m, 0}+w_{m, 0}\right|^{2}+\alpha}{E\left\{x_{m, 0}^{2}\right\}}-\left|H_{m, 0}\right|^{2}\right\} \\
& =\frac{\overbrace{E\left\{2 \Re \mathfrak{R e}\left(x_{m, 0} H_{m, 0} I_{m, 0}^{*}\right)\right\}}^{\sigma_{H I}^{2}}+\sigma^{2}+\alpha}{E\left\{x_{m, 0}^{2}\right\}} \\
& =\frac{\sigma^{2}+\alpha}{E\left\{x_{m, 0}^{2}\right\}},
\end{aligned}
$$

since $\mathfrak{R e}\left(x_{m, 0}\right)=x_{m, 0}$ is a real zero-mean variable, which is independent of $H_{m, 0}$ and $I_{m, 0}$. Thus, a qualitative comparison with the solution $\left|F_{m, 0}\right|<<1$ can be made. In fact, it should be noticed that $\epsilon_{F}>>1$ if $\left|F_{m, 0}\right|<<1$ is substituted in (28) instead of $F_{m, 0}^{s-o p t}$, whereas the error $\epsilon_{F}$ using the proposed initialization in (29) is inversely proportional to the symbol power to noise power ratio. Therefore, we conclude that the sub-optimal $F_{m, 0}^{s-o p t}$ solution in (27) should achieve better performance than $\left|F_{m, 0}\right|<<1$. This behavior will be numerically verified in Section IV. Furthermore, the computation of $F_{m, 0}^{s-o p t}$ in (27) requires one multiplication for $\left|y_{m, 0}\right|^{2}$, one addition, and one division. The value of $E\left\{x_{m, 0}^{2}\right\}$ only depends on the constellation size, and therefore it can be computed off-line.

\section{Simulations Results}

In this section, simulations results show the performance of the different blind equalization methods proposed in this paper. 


\section{A. Simulations Parameters}

Since we compare the solution proposed in Sections III with the CMA in [11] and [12], the same simulation parameters described in [11] are considered. The path gains of the constant multipath channel are $\mathbf{h}=[1,-0.2,0.3,0.2,0.1,0.2,0.35,-0.2]$, and the phase of the channel is uniformly chosen in $\left[-\frac{\pi}{2}, \frac{\pi}{2}\right]$. Each transmitted OFDM/OQAM symbol is composed of $M=128$ subcarriers of $15 \mathrm{kHz}$ width. The system operates at a carrier frequency of $400 \mathrm{MHz}$. According to [11] the constant initialization is set equal to $F_{m, 0}=0.01$ for any $m \in\{0,1, . ., M-1\}$. In order to avoid the divergence of the algorithm when the proposed initialization $F_{m, 0}^{s-o p t}$ is used, we take $\alpha=0.01$. Furthermore, a 16-QAM constellation is used, i.e. OQAM symbols are randomly chosen among $\{-3,-1,1,3\}$, and the CMA in (13) is carried out with $p=2$ and $\mu=0.0033$. This configuration is also used to test the equalizer using the CNA-6 in OFDM/OQAM.

The performance of the different blind equalizers is assessed by using the mean square error MSE defined as

$$
\operatorname{MSE}=E\left\{\left(\left|a_{m, n}\right|-\left|x_{m, n}\right|\right)^{2}\right\} .
$$

The achieved MSEs of the blind equalizers are also compared with that of the data-aided estimation using interference approximation method (IAM) proposed in [4]. The preamble of IAM is designed as follows: for any pilot $x_{m, n}= \pm \mathcal{P}$, then the symbols at positions $n-1$ and $n+1$ are zero, and $x_{m-1, n}=-x_{m+1, n}$. Therefore the transmultiplexer gain described in (5) yields a received pilot with an energy larger than $|\mathcal{P}|^{2}$. This property makes IAM one of the most robust channel estimator in OFDM/OQAM, and we denote by $\hat{H}_{m, n}^{I A M}$ the estimated channel coefficients. The channel is then inverted with the zero-forcing (ZF) equalizer such as $F_{m, n}^{I A M}=1 / \hat{H}_{m, n}^{I A M}$. Note that the energy of the pilot is the same as the transmitted symbols, i.e. $|\mathcal{P}|^{2}=5$. It must be emphasized that the results presented for the estimator using IAM are only indicative. In fact, a fair comparison would induce to test different channel estimation methods (IAM-R [6], MMSE [20]), pilot energy, or equalizers [8]. A more exhaustive comparison with pilot-aided methods is out of the scope of this paper, but 
numerous estimation and equalization techniques can be found [6], [8], [20].

\section{B. Performance of CNA-6 in OFDM/OQAM}

First series of simulations have been performed to analyze the performance of blind equalization using CNA-6 in OFDM/OQAM. Fig. 6 shows the trajectories of the CMA and the CNA-6 versus the OFDM/OQAM symbols, for a signal-to-noise ratio (SNR) of $30 \mathrm{~dB}$. Two initialization values $F_{m, 0}=0.01$ and $F_{m, 0}^{s-o p t}$ have been tested. Furthermore, the achieved MSE of IAM is given as reference. It can be seen that the steady-state performance of CNA-6 has 1 dB gain compared with the MSE floor using CMA. This result is consistent with the analysis undertaken in Section III-C. However, the CNA-6 using $F_{m, 0}=0.01$ requires at least 1000 OFDM/OQAM symbols more than CMA to achieve a given MSE value. This loss is reduced by half when the algorithms are initialized with $F_{m, 0}^{s-o p t}$. This delay in the convergence is due to the intrinsic phase adjustment when using CNA6. The basic idea behind this phenomenon can be described by using the balls of the equalizers in Fig. 3. In fact, it can be observed in Fig. 3-(a) that CMA is phase-independent since the balls of CMA is a circle. However, the optimal equalizer coefficient value depends on the phase of $F_{m, n}$ (see Fig. 3-(b)), therefore the phase adjustment slows down the convergence of CNA-6. This phenomenon has been described in detail in [15]. It is also worth noticing that the use of the sub-optimal $F_{m, 0}^{s-o p t}$ value allows the algorithm to gain 2000 iterations compared with $F_{m, 0}=0.01$ for both CMA and CNA-6. Finally, Fig. 6 highlights that a gain of $1 \mathrm{~dB}$ and $2 \mathrm{~dB}$ is achieved by CMA and CNA-6 respectively compared with IAM. This shows the capability of the blind equalization to outperform data-aided techniques.

Fig. 7 depicts the MSE-floor values of CMA, CNA-6, and IAM when the steady-state is reached (at the 3000-th iteration) versus SNR. It can be observed that the blind equalizers achieved an MSE gain (up to $12 \mathrm{~dB}$ at $\mathrm{SNR}=5 \mathrm{~dB}$ ) compared with IAM for SNR values $<30 \mathrm{~dB}$. This reflects the fact that the updated coefficients $F_{m, n}$ in (13) or (22) are less disturbed in noisy conditions than the ZF coefficient $F_{m, n}^{I A M}$ using IAM. However, it is worth noticing that these results are obtained after 3000 iterations, whereas no convergence delay is required for data-aided methods (see Fig. 6 for instance). 


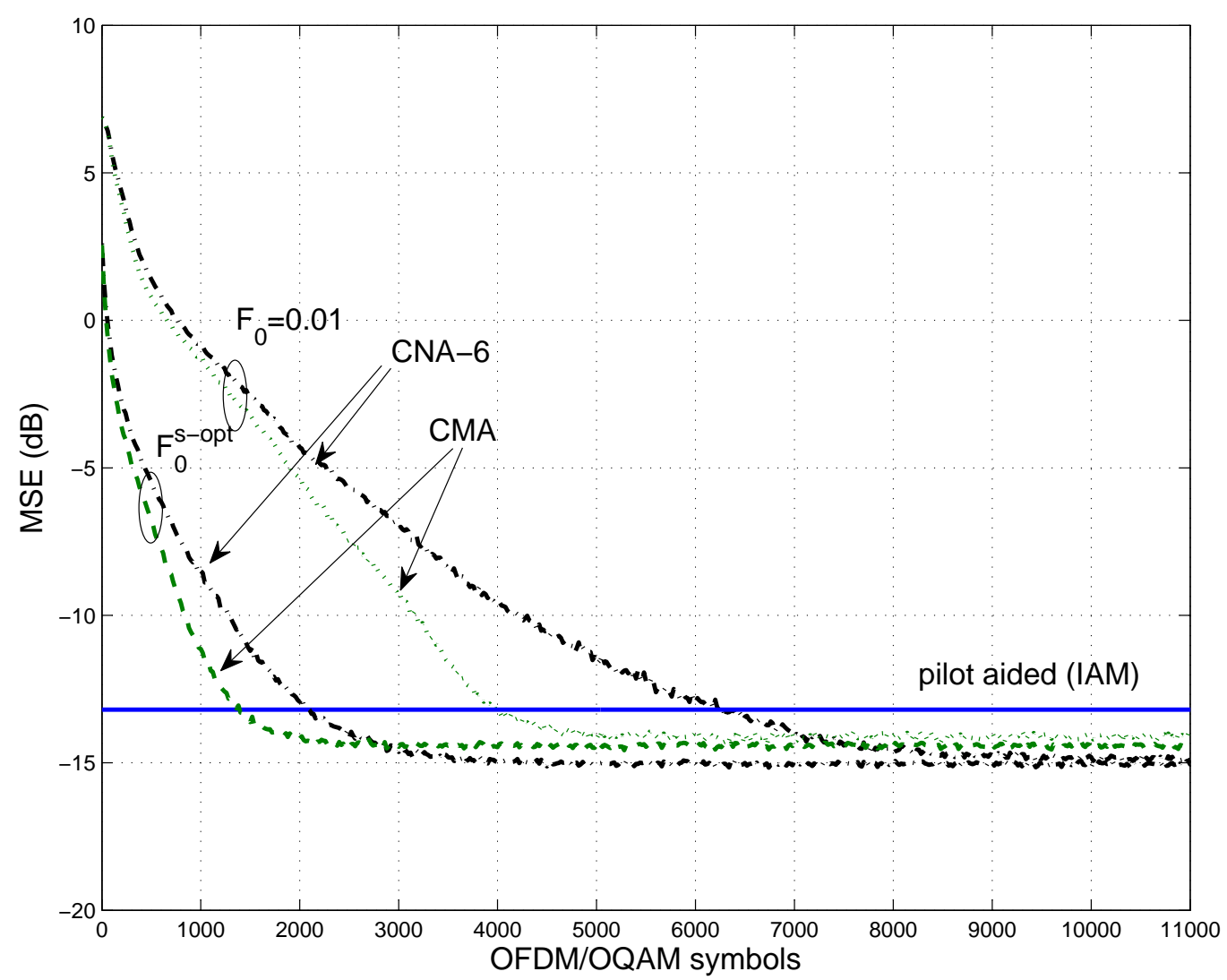

Fig. 6. MSE performance versus OFDM/OQAM symbols. Comparison between CNA-6, CNA-2 (CMA) using $F_{m, 0}=0.01$ and $F_{m, 0}^{s-o p t}$, and pilot-aided method (IAM).

Furthermore, the MSE value of the IAM also depends on the pilot energy $|\mathcal{P}|^{2}$. Otherwise, it can be also observed that CNA-6 achieves better performance than CMA for SNR $>20 \mathrm{~dB}$.

Fig. 8 presents the results of simulations undertaken to compare the convergence speed of CMA and CNA- 6 for a given steady-state performance. Thus, the $\mu$ values have been tuned so that the MSE floor values of both equalizers reach the same value at $-16.5 \mathrm{~dB}$. It can be observed that CNA-6 converges faster than CMA, e.g. a gain of 1200 iterations is achieved using CNA- 6 at MSE=-13.5 $\mathrm{dB}$ (which corresponds to the MSE of estimation using IAM) compared with CMA. We conclude from Figs. 6 and 8 that, for a given convergence rate (i.e. for the same $\mu$ value) the CNA-6 achieves lower MSE than CMA, and for a given steady-state the CNA-6 converges faster than CMA. 


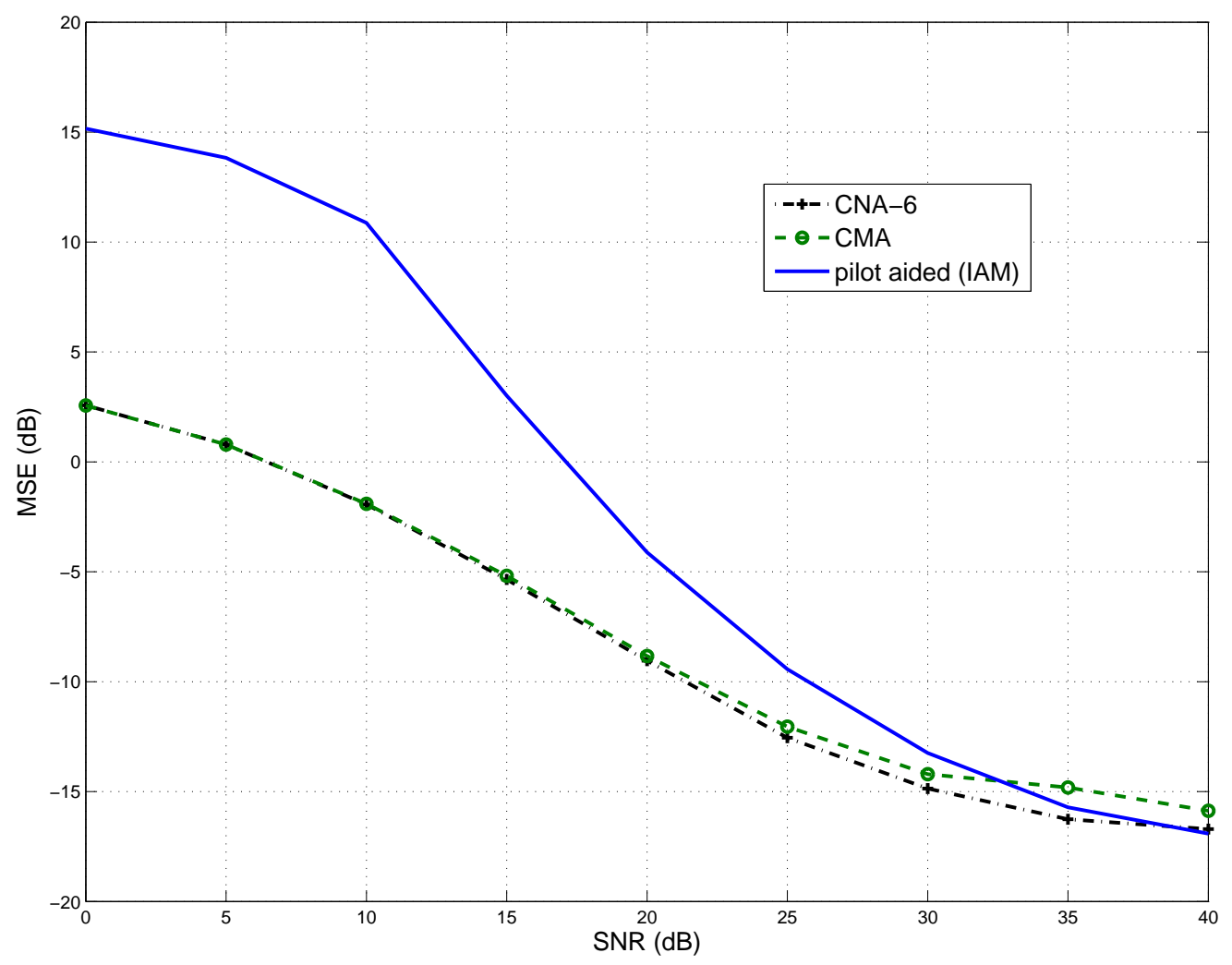

Fig. 7. MSE performance versus SNR. Comparison between CNA-6, CNA-2 (CMA) at the 3000-th iteration, and pilot-aided method.

As indicated in Section II, the blind receiver may suffer from synchronization mismatch. The effect of such a time synchronization mismatch on the performance of CMA and CNA-6 is depicted in Fig. 9. The time shift between the transmitter and the receiver is given as a percentage of the prototype filter length $L_{f}$. The performance of the system using IAM is shown as well, but it is only indicative. It can be observed that time synchronization mismatches of 1\%,3\%, and 5\% induce MSE losses of 11, 13, and $16.5 \mathrm{~dB}$, respectively, compared with the results given in Fig. 6. Such a MSE loss is due to the interferences induced by the synchronization mismatch. Furthermore, additional simulations revealed that a time shift larger than 5\% leads to instability, i.e. the blind equalizer diverges. These results show that both CMA and CNA-6 require a very accurate synchronization process in order to work at their best performance. 


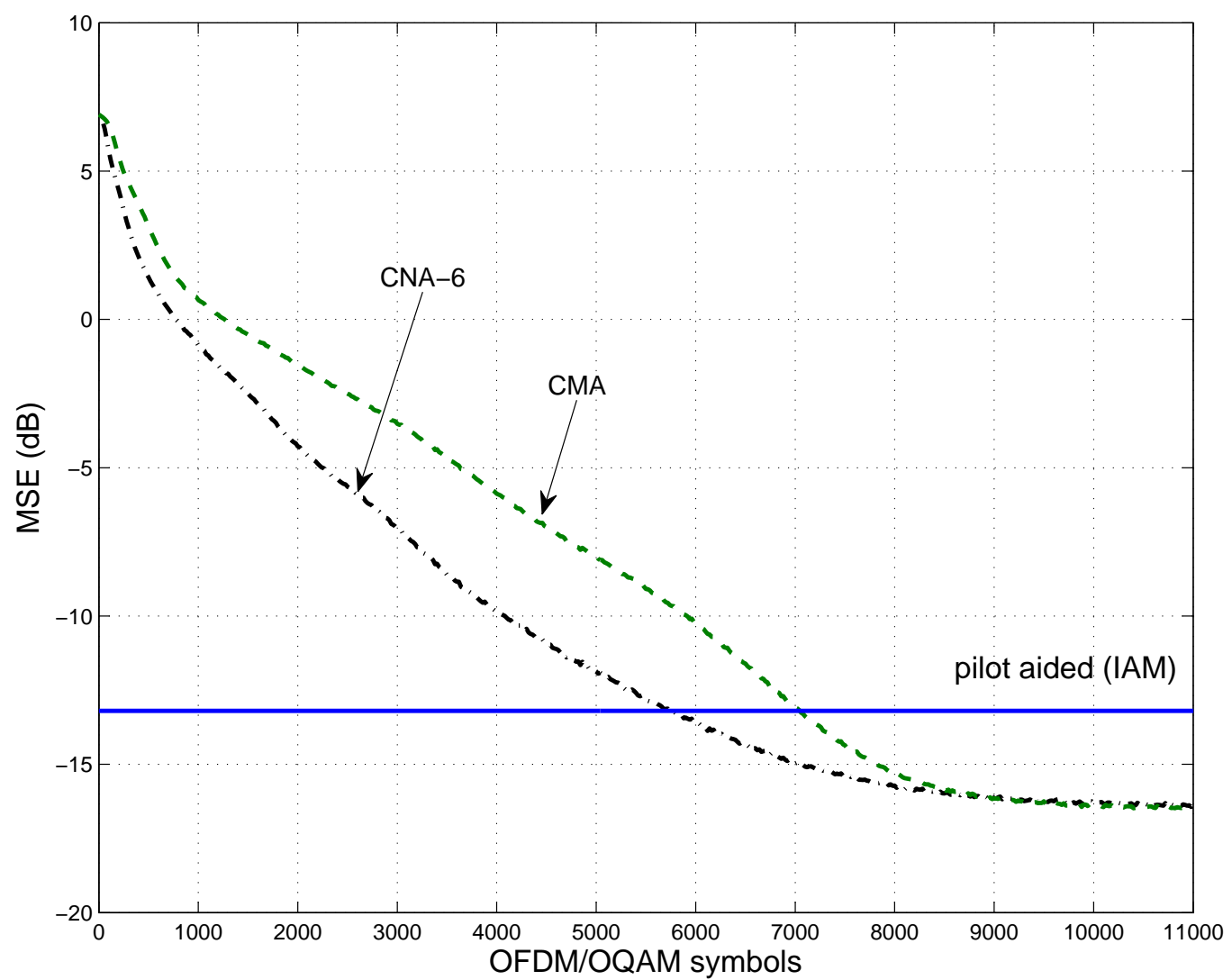

Fig. 8. MSE performance versus OFDM/OQAM symbols. Comparison between CNA-6, CNA-2 (CMA), and pilot-aided method.

Fig. 10 shows the MSE performance of CNA-6, CNA-2 (CMA) versus OFDM/OQAM symbols for three receiver speeds: (a) $1 \mathrm{~km} / \mathrm{h}$, (b) $3 \mathrm{~km} / \mathrm{h}$, and (c) $10 \mathrm{~km} / \mathrm{h}$. Two different initialization strategies have been considered: i) using $F_{m, 0}^{s-o p t}$, and ii) using a preamble such that $F_{m, 0}=1 / \hat{H}_{m, n}^{I A M}$ (referred to "pilot init." on the figures). It can be observed that the equalizers performances tend to the MSE level whatever the initialization is (for $1 \mathrm{~km} / \mathrm{h}$, this limit is outside the OFDM/OQAM symbols range). However, the MSE starts at $4 \mathrm{~dB}$ where $F_{m, 0}^{s-o p t}$ is used, whereas it starts at -8.5 $\mathrm{dB}$ when $F_{m, 0}=1 / \hat{H}_{m, n}^{I A M}$ is used. Thus, it can be noted that the CMA and CNA-6 equalizer using $F_{m, 0}=1 / \hat{H}_{m, n}^{I A M}$ outperforms those using $F_{m, 0}^{s-o p t}$ from 0 to 5000 iterations and from 0 to 2500 iteration for $3 \mathrm{~km} / \mathrm{h}$ and $10 \mathrm{~km} / \mathrm{h}$ respectively. Furthermore, it can be also noted in (a) and (b) that 


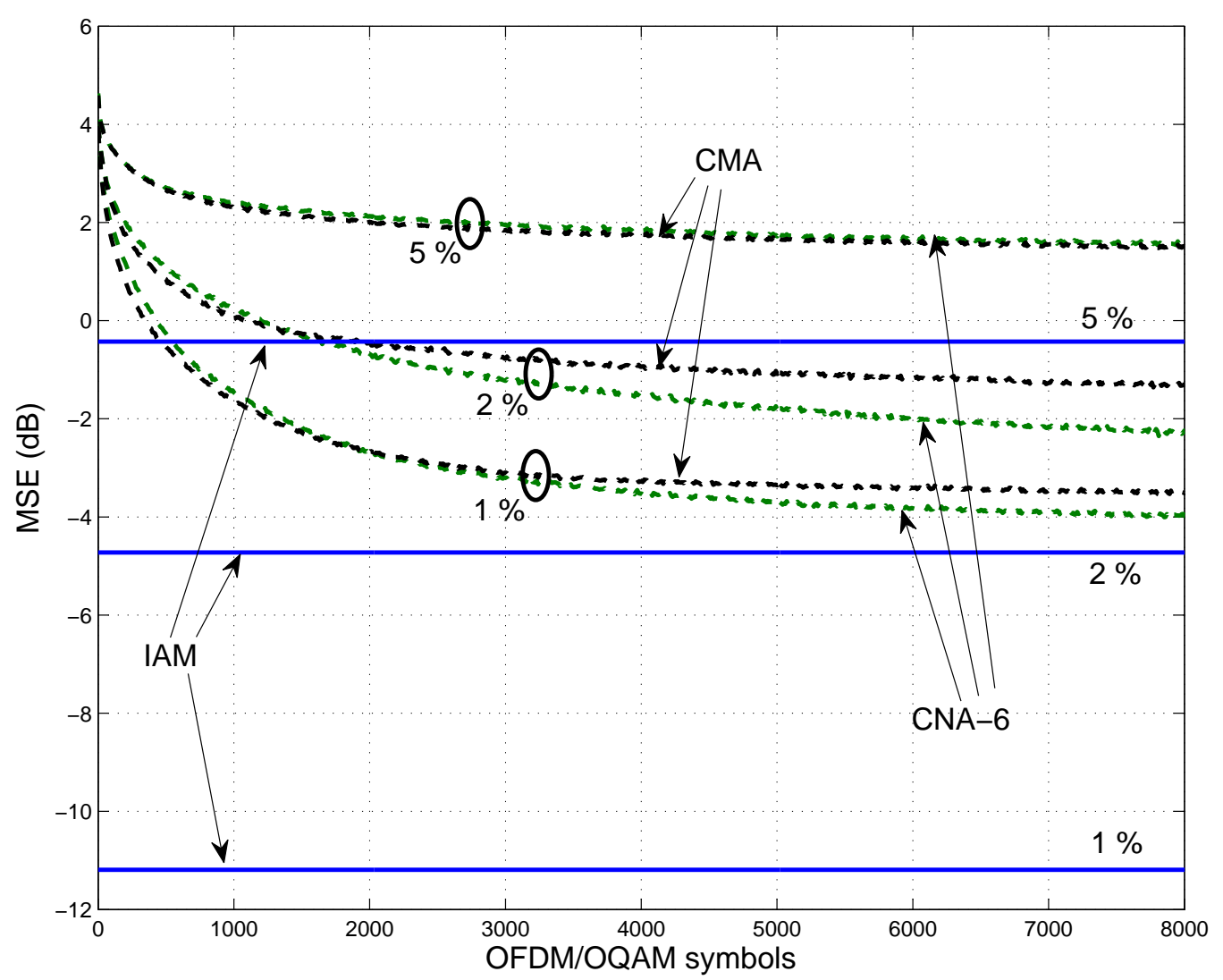

Fig. 9. MSE performance of CNA-6, CNA-2 (CMA), and pilot-aided method versus OFDM/OQAM symbols in presence of time synchronization mismatch. The synchronization mismatch is given as a percentage of $L_{f}=\{1 \%, 2 \%, 5 \%$.

CMA achieves 0.5 MSE gain compared with CNA-6 where the steady state is reached the steady-state. This is mainly due to the approximation in (16), which does not hold anymore when time selectivity of the channel increases.

\section{Discussion}

The practical use of blind equalizers such as CMA and CNA-6 is discussed in this section. We deduce from Figs. 6 and 8 that, even if the use of $F_{m, 0}^{s-o p t}$ and CNA-6 reduces the convergence delay, blind equalizers still require more than 1000 OFDM/OQAM symbols to converge. As a consequence, the use of these methods requires is limited by the length of the signal packets. Thus, broadcast applications could be a possible context to operate blind receivers. Note that the reuse of the received 


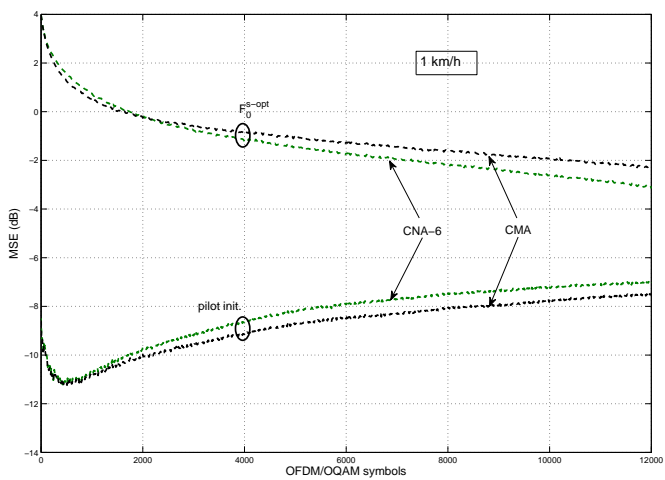

(a) $1 \mathrm{~km} / \mathrm{h}$ receiver speed.

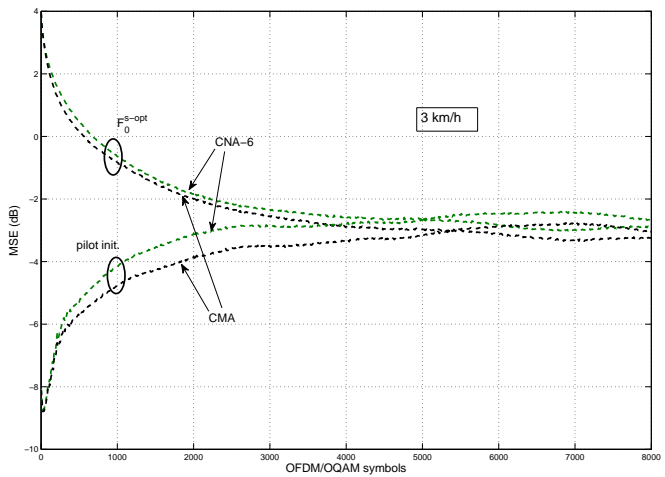

(b) $3 \mathrm{~km} / \mathrm{h}$ receiver speed.

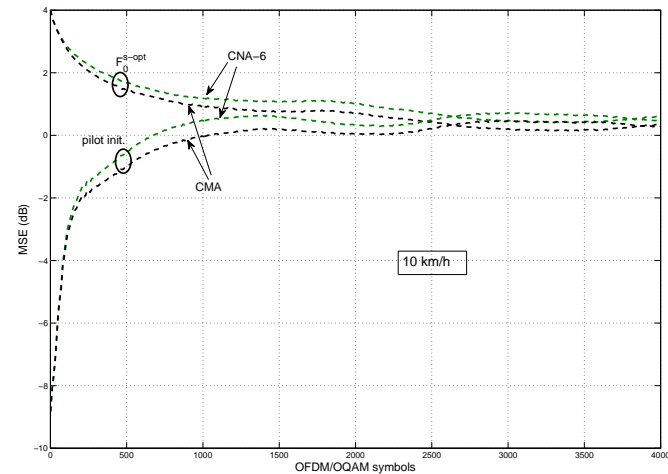

(c) $10 \mathrm{~km} / \mathrm{h}$ receiver speed.

Fig. 10. MSE performance of CNA-6, CNA-2 (CMA) versus OFDM/OQAM symbols using the initialization $F_{m, 0}^{s-o p t}$ compared with the initialization using a preamble, for three receiver speeds: (a) $1 \mathrm{~km} / \mathrm{h}$, (b) $3 \mathrm{~km} / \mathrm{h}$, and (c) $10 \mathrm{~km} / \mathrm{h}$. 
symbols as input of the equalizers seems to be a good solution to further reduce the convergence delay [29].

It has been shown in Fig. 9 that the blind equalizers are very sensitive to time synchronization mismatch. Therefore, an accurate synchronization process is required. A usual solution is to undertake this task thanks to a preamble [22]. Furthermore, this preamble could be used for channel estimation. Fig. 10 revealed that an initialization of the blind equalizers thanks to an accurate estimate of the channel allows to hold lower MSE values much more longer than when using $F_{m, 0}^{s-o p t}$ in slowly time-varying environments. Thus, instead of using full-blind equalizers as in Figs. 6 and 8, one can deduce from Figs. 9 and 10 that the use of regular preamble (e.g. at the beginning of each frame) could tackle the issue of synchronization mismatch, as well as allowing blind equalizers to operate in weakly time-selective channels.

\section{CONCLUSion}

In this paper, we have presented an adaptation of the CNA blind equalizer for OFDM/OQAM modulation. In Section III, the CNA, which can be seen as a generalized CMA, has been developed. To be effective, the CNA requires a signal using a complex constellation. Therefore, it has been proposed to reshape the "real" received OQAM symbols to obtain "complex" 16-QAM symbols. The performance of the proposed method has been analyzed, and it has been proved that, despite the increase of the interference level due to the reshape, the CNA applied to the new signal achieves better performance than the CMA applied to the OFDM/OQAM signal. Furthermore, the principle of changing the shape of the received signal opens new perspectives for the blind equalization in the FBMC-based modulations, as specific cost functions (not limited to the real field) can now be investigated. In addition, a sub-optimal initialization strategy, in the sense of the mean square error, has been presented. Numerical results revealed that the CNA and the proposed initialization method allows to reduce by 2000 the number of iterations of the blind equalizer. Moreover, the performances of CMA and CNA-6 have been studied in conditions where the receiver is not well-synchronized with the transmitter and the channel is time-varying. Results have shown that this degrades the performance. 
In order to overcome these deteriorations, a relevant solution consists of using preamble, from which the synchronization and accurate initialization can be obtained.

\section{APPENDIX}

A. Obtaining the Average Distances $E\left\{l_{6}\right\}$ and $E\left\{l_{2}\right\}$

The ball corresponding to the CMA is generated by $\left|a_{m, n}\right|^{2}-\gamma=0$, i.e. it is a circle of radius $\sqrt{\gamma}=\sqrt{8.2}$. Since $\mathbb{P}\left(x_{m, n}= \pm 1\right)=\mathbb{P}\left(x_{m, n}= \pm 3\right)=0.5$, the average distance $E\left\{l_{2}\right\}$ can be expressed as

$$
\begin{aligned}
E\left\{l_{2}\right\} & =\mathbb{P}\left(x_{m, n}= \pm 1\right)(\sqrt{\gamma}-1)+\mathbb{P}\left(x_{m, n}= \pm 3\right)(3-\sqrt{\gamma}) \\
& =1
\end{aligned}
$$

The ball corresponding to the CNA-6 is defined by $\left\|z_{m, n}\right\|_{6}^{2}-\gamma=0$, and it is depicted in Fig. 3-(b). The radius of this ball along the $X$ axis is $\sqrt{\gamma}=\sqrt{13.2}$, while its radius along the axis $y=x$ is given by $\frac{\sqrt{2 \gamma}}{2^{1 / 6}}$. Since $\mathbb{P}\left(x_{m, n}= \pm 1 \pm j\right)=\mathbb{P}\left(x_{m, n}= \pm 3 \pm 3 j\right)=\mathbb{P}\left(x_{m, n}= \pm 1 \pm 3 j\right)=\mathbb{P}\left(x_{m, n}=\right.$ $\pm 3 \pm j)=0.25$, the average distance $E\left\{l_{6}\right\}$ can be expressed as

$$
\begin{aligned}
E\left\{l_{6}\right\} & =\mathbb{P}\left(x_{m, n}= \pm 1 \pm j\right)(\sqrt{\gamma}-1) \\
& +\mathbb{P}\left(x_{m, n}= \pm 3 \pm 3 j\right)\left(\sqrt{18}-\frac{\sqrt{2 \gamma}}{2^{1 / 6}}\right) \\
& +\mathbb{P}\left(x_{m, n}= \pm 1 \pm 3 j\right)(\sqrt{\gamma}-3) \\
& +\mathbb{P}\left(x_{m, n}= \pm 3 \pm j\right)(\sqrt{\gamma}-3) \\
& =1.0586
\end{aligned}
$$

\section{REFERENCES}

[1] B. L. Floch, M. Alard, and C. Berrou, "Coded Orthogonal Frequency Division Multiplex," Proceedings of the IEEE, vol. 83, no. 6, pp. 982-996, June 1995. 
[2] B. Farhang-Boroujeny, “OFDM Versus Filter Bank Multicarrier,” IEEE Signal Processing Magazine, vol. 28, no. 3, pp. 92 - 112, May 2011.

[3] J.-P. Javaudin, D. Lacroix, and A. Rouxel, "Pilot-Aided Channel Estimation for OFDM/OQAM," in proc. of VTC'03 Spring, vol. 3, Jeju, Korea, April 2003, pp. 1581 - 1585.

[4] C. Lélé, J.-P. Javaudin, R. Legouable, A. Skrzypczak, and P. Siohan, "Channel estimation methods for preamble-based OFDM/OQAM modulations," European Transactions on Telecommunications, vol. 19, no. 7, pp. 741 -750, November 2008.

[5] D. Katselis, E. Kofidis, A. Rontogiannis, and S. Theodoris, "Preamble-Based Channel Estimation for CP-OFDM and OFDM/OQAM Systems: A Comparative Study," IEEE Transactions on Signal Processing, vol. 58, no. 5, pp. 2911 2916, february 2010.

[6] E. Kofidis, D. Katselis, A.Rontogiannis, and S. Theodoridis, "Preamble-based channel estimation in OFDM/OQAM systems: A review," Signal Processing, Elsevier, vol. 93, pp. 2038 - 2054, January 2013.

[7] G. Lin, L. Lundheim, and N. Holte, "On efficient equalization for OFDM/OQAM systems," in proc. of InOWo'05, Hamburg, Germany, 31 August - 1 September 2005, pp. $1-5$.

[8] T. Ihalainen, T. H. Stitz, M. Rinne, and M. Renfors, "Channel Equalization in Filter Bank Based Multicarrier Modulation for Wireless Communications," EURASIP Journal on Advances in Signal Processing, vol. 2007, pp. $1-18$, August 2007.

[9] T. Ihalainen, A. Ikhlef, J. Louveaux, and M. Renfors, "Channel Equalization for Multi-Antenna FBMC/OQAM Receivers,” IEEE Transactions on Vehicular Technology, vol. 60, no. 5, pp. 2070 - 2085, April 2011.

[10] H. Bölcskei, P. Duhamel, and R. Hleiss, “A Subspace-Based Approach to Blind Channel Identification in Pulse Shaping OFDM/OQAM Systems," IEEE Transactions on Signal Processing, vol. 49, no. 7, pp. 1594 - 1598, July 2001.

[11] B. Farhang-Boroujeny, "Multicarrier Modulation With Blind Detection Capability Using Cosine Modulated Filter Banks," IEEE Transactions on Communications, vol. 51, no. 12, pp. 2057 - 2070, December 2003.

[12] L. Lin and B. Farhang-Boroujeny, "Convergence analysis of blind equalizer in a cosine modulated filter bank-based multicarrier communication system," in proc. of SPAWC'03, Roma, Italy, June 2003, pp. 368 - 372.

[13] Y. Sato, “A Method of Self-Recovering Equalization for Multilevel Amplitude-Modulation Systems," IEEE Transactions on Communications, vol. 23, no. 6, pp. 679 - 682, June 1975.

[14] J. Yang, J.-J. Werner, and G. A. Dumont, "The Multimodulus Blind Equalization and Its Generalized Algorithms," IEEE Transactions on Selected Areas in Communications, vol. 20, no. 5, pp. 997 - 1015, June 2002.

[15] A. Goupil and J. Palicot, "New Algorithms for Blind Equalization: The Constant Norm Algorithm Family," IEEE Transactions on Signal Processing, vol. 55, no. 4, pp. 1436 - 1444, April 2007.

[16] K. N. Oh and Y. O. Chin, "Modified constant modulus algorithm: blind equalization and carrier phase recovery algorithm," in proc. of ICC'95, vol. 1, Seattle, WA, June 1995, pp. 498 - 502. 
[17] S. Barbarossa, "Blind Equalization Using Cost Function Matched to the Signal Constellation," in proc. of ACSSC'97, vol. 1, Pacific Grove, CA, USA, November 1997, pp. 550 - 554.

[18] P. Siohan, C. Siclet, and N. Lacaille, “Analysis and Design of OFDM/OQAM Systems Based on Filterbank Theory,” IEEE Transactions on Signal Processing, vol. 50, no. 05, pp. 1170 - 1183, May 2002.

[19] M. Bellanger, "Specification and Design of a Prototype Filter for Filter Bank Based Multicarrier Transmission," in proc. of ICASSP'01, vol. 4, Salt Lake City, UT, May 2001, pp. 2417 - 2420.

[20] V. Savaux, F. Bader, and Y. Louët, "A Joint MMSE Channel and Noise Variance Estimation for OFDM/OQAM Modulation,” IEEE Transactions on Communications, vol. 63, no. 11, pp. 4254 - 4266, November 2015.

[21] Y. Medjahdi, M. Terre, D. Le Ruyet, D. Roviras, and A. Dziri, “The Impact of Timing Synchronization Errors on the Performance of OFDM/FBMC Systems,” in proc. of ICC'11, Kyoto, Japan, June 2011, pp. 1 - 5.

[22] N. Cassiau, D. Kténas, and J.-B. Doré, "Time and frequency synchronization for CoMP with FBMC," in proc. of ISWCS'13, Ilmenau, Germany, August 2013, pp. 1 - 5.

[23] Y. Zeng and M. W. Chia, "Joint Time-Frequency Synchronization and Channel Estimation for FBMC," in proc. of PIMRC'14, Washington DC, DC, USA, September 2014, pp. 1 - 5.

[24] D. N. Godard, "Self-Recovering Equalization and Carrier Tracking in Two-Dimensional Data Communication Systems," IEEE Transactions on Communications, vol. com-28, no. 11, pp. 1867 - 1875, November 1980.

[25] R. Johnson, Jr, P. Schniter, T. Endres, J. Behm, D. Brown, and R. Casas, "Blind Equalization Using the Constant Modulus Criterion: A Review,” Proceedings of the IEEE, vol. 86, no. 10, pp. 1927 - 1950, October 1998.

[26] P. Bouboulis, “Wirtingers Calculus in general Hilbert Spaces," ArXiv, vol. arXiv:1005.5170v1, pp. 1 - 27, May 2010.

[27] A. Goupil and J. Palicot, "Constant norm algorithms class," in proc. of EUSIPCO, Toulouse, France, September 2002.

[28] V. Savaux and F. Bader, "Sub-Optimal Initialization for Blind Equalization with Fast Convergence in OFDM/OQAM Modulation," in proc. of EuCNC'15, Paris, France, June - July 2015.

[29] V. Savaux, M. Naoues, F. Bader, and J. Palicot, "Ultra-Fast Blind Equalization for OFDM: Principle and Steps Towards Implementation," in proc. of ISWCS'15, Brussels, Belgium, August 2015, pp. 1 - 5. 
PLACE

Faouzi Bader (SM07) received the Ph.D. degree (with Honours) in telecommunications from Universidad Politcnica de Madrid (UPM), Madrid, Spain, in 2002. He joined the Centre Technologic de

HERE Telecomunicacions de Catalunya (CTTC), Barcelona, Spain, as Associate Researcher, in 2002, and was nominated in 2006 as a Senior Research Associate at same institution. Since June 2013, he has worked as an Associate Professor at cole Suprieur dElectricitSUPELEC in Rennes, France. His research activities mainly focus on IMT-Advanced systems, system design, advanced multicarrier waveforms (OFDM(A), (non)uniform multimode filter based multicarrier schemes), and frequency allocation techniques in relay cognitive environment. He has been involved in several European projects from the 5th7th EC research frameworks, and from 2012 to 2013 he was nominated the General Coordinator and Manager of the EC funded research project ICT EMPhAtiC project, Enhanced Multicarrier Techniques for Professional Ad-Hoc and Cell-Based Communications. He has published over 120 papers in peer-reviewed journals and international conferences, more than 13 book chapters, and 3 books. He served as Technical Program Committee member in major IEEE ComSoc and VTS conferences, and as the General Chair of the 11th edition of the ISWCS2014 conference, and the Cochair of the ISWCS 2015 edition. 
Jacques Palicot He received, in 1983, his PhD degree in Signal Processing from the University

PHOTO of Rennes. Since 1988, he has been involved in studies about equalization techniques applied to

HERE digital transmissions and analog TV systems. Since 1991 he has been involved mainly in studies concerning the digital communications area and automatic measurements techniques. He has taken an active part in various international bodies EBU, CCIR, URSI, and within RACE, ACTS and

IST European projects. He has published various scientific articles notably on equalization techniques, echo cancellation, hierarchical modulations and Software Radio techniques. He is author or co-author of more than 300 publications with more than 50 in prestigious journals, two books and 22 patents. He is currently involved in adaptive Signal Processing, digital communications, Software Radio, Cognitive radio and Green Radio. From November 2001 to September 2003 he had a temporary position with INRIA/IRISA in Rennes. He serves as Associate Editor for EURASIP JASP since 2008. He also served as lead guest editor for several Special Issues on Software Radio, Cognitive Radio and Green Radio. He was Co General Chairman of ISCIT 2011, Co General Chairman of Next-GWiN 2014, Technical Program Chairman of CROWNCOM 2009, Technical Program Chairman of GREENCOM 2013 and Technical Program Chairman of CRN Symposium of ICC 2014. Since October 2003 he is with CentraleSuplec in Rennes where he leads the Signal Communications and Embedded Electronics (SCEE) research team. 